Research Article

\title{
Raking Effects on Aggregate Densification and Channelling in Thickening
}

\author{
Xu Zhou $\mathbb{D}^{1},{ }^{1,2}$ Hong-jiang Wang $\mathbb{D}^{1},{ }^{1}$ Hua-zhe Jiao $\mathbb{D}^{\circ},{ }^{3}$ and Liu-hua Yang $\mathbb{D}^{1}$ \\ ${ }^{1}$ School of Civil and Engineering, University of Science and Technology Beijing, Beijing 100083, China \\ ${ }^{2}$ Xinda Gold and Silver Development Center Ltd., Beijing 100038, China \\ ${ }^{3}$ School of Civil Engineering, Henan Polytechnic University, Henan 454003, China \\ Correspondence should be addressed to Xu Zhou; pedrozhxu@gmail.com, Hong-jiang Wang; wanghj1988@126.com, \\ and Liu-hua Yang; yanglh2005@163.com
}

Received 13 February 2019; Accepted 28 March 2019; Published 27 June 2019

Academic Editor: Joon-Hyung Lee

Copyright (c) 2019 Xu Zhou et al. This is an open access article distributed under the Creative Commons Attribution License, which permits unrestricted use, distribution, and reproduction in any medium, provided the original work is properly cited.

\begin{abstract}
Thickening performance is greatly influenced by aggregate densification and channelling development; however, the microstructures of aggregates and channelling are unable to directly observe because of high turbidity mud bed during thickening. Computed tomography scan imaging technique has been introduced to investigate the effects of aggregate densification and channelling in the mud bed samples during unclassified tailings thickening. The samples were prepared through deep-freezing and freeze-drying techniques, immediately after sampling from the operation of a pilot thickener. Based on the information of void ratio, pore-size distribution, and permeability, obtained from 3D reconstruction images of the aggregates structure, aggregate densification and channelling development have been characterized. Channelling patterns have been classified by the size and shape of the connection throat of the pore between aggregates. As the aggregate structure broke and densified, the void ratio and pore size of the mud bed were decreased. Thickening performance enhancement was predicted based on solid flux and permeability of mud bed, and the result indicated raking on aggregate densification and channelling apparently improves the unclassified tailings dewatering extent.
\end{abstract}

\section{Introduction}

Tailings are the waste material residuals after metals have been extracted from ore by physical and chemical techniques [1]. As the traditional tailings disposal, low-concentration tailings slurry was discharged and stored in tailings storage facility on the surface directly. It requires less equipment expenditure, but large footprint of tailings dam and high construction cost; the risk of tailing dam failure and environment impact cannot also be ignored [2]. One of the solutions is the use of cement paste backfill (CPB) as artificial pillars or stable platform for mining operation, to ensure the stability of the underground excavations and decrease the quantity of tailings stored on the surface during mine operations $[3,4]$, as well as reduce the potential of tailing dam failure and surface subsidence disaster [5]. CPB is composed of thickened unclassified mill tailings $(70-85 \%$ solid dry mass by weight), which is mixed with small proportion of hydraulic binder (3-7wt.\%) such as Portland cement, fly ash, or smelter slag[6]. After sufficient mixing, this high density non-Newtonian mixture was placed into the underground voids or mined stopes through pipe line by pump or gravity flow [7]. The greatest challenge is to make the $\mathrm{CPB}$ with enough strength and proper pump ability at minimum operation cost, which requires the concentration of dewatered tailings slurry under accurate prediction and control.

Based on gravity sedimentation technology, paste thickener provides a highly efficient and low-cost tailings dewatering solution for $\mathrm{CPB}$ preparation [8]. In order to accurately control solid concentration and stability of underflow tailings slurry, dewatering theory mainly focused on the rheological characteristics during thickening, such as the hinder settling function and compressive yield stress $[9,10]$; however, the microstructure of aggregate densification and channelling development of mud bed slurry under raking condition also needs further investigation. 
Channelling or fingering flow randomly occurred in selfgravity consolidation of fine-particle solids suspension, which affects the consolidation and drainage extent $[11,12]$. Meanwhile, the mechanics of channelling and the effects of channelling on tailings sedimentation or thickener dewatering are highly important. Channelling has been observed from batch sedimentation trials with various materials, flocculants, and initial concentrations [13]. During batch settling and compressive consolidation process of thickening, channelling presents as a microstructure of narrow paths through the mud bed; water and fine solid particles escaped upwards through low concentration voids or channels, thus creating small amounts of accumulated materials such as volcanoes or small depressions of craters on the interface of water and solid [14]. Holdich and Butt [15] studied compression with "soft and hard channel" formation in batch sedimentations of mineral talc suspended in water at various initial concentrations and provided a numerical model for compressible sedimentation with channel. Glasrud et al. [16] detected channelling accelerated settling ratio during settling behaviours of flocculated magnetic and nonmagnetic iron-oxide suspensions. Nam et al. [17] observed channel formation in settling columns with different materials and initial concentration, and the results indicated that the channel formation was more prevalent with a stronger degree of flocculation. Channelling has been observed in batch settling or sedimentation test, although the mechanism of channel formation and aggregate structure deformation under raking in paste thickener and the effects of raking on aggregate densification and channelling during paste thickening are still not fully understood. For this purpose, this paper carried out a study on aggregate and channelling features through CT scanning images; furthermore, raking effects on aggregate densification and channelling development in thickening have been analysed.

\section{Materials and Methods}

2.1. Pilot Thickener System. The continuous pilot thickener system (Figure 1) consisted of a pilot thickener column, a feed system of tailings slurry and polymer solution, and a discharge system of overflow and underflow. The pilot thickener column is $15 \mathrm{~cm}$ in internal diameter with $100 \mathrm{~cm}$ in height, which is made up of 5 sections of $20 \mathrm{~cm}$ height Perspex cylinders. An overhead-geared motor-driven rotating picket rake was set up by using a steel shaft with 4 vertical rods. The tailings slurry and polymer solution were fed by peristaltic pumps through branch feed tubes from holding tanks individually. Tailings slurry and polymer solution were mixed in the main feeding tube and then flowed into the thickener. During the pilot thickener system operation, supernatant water was collected from the overflow outlet and high-concentration tailings underflow was discharged from the bottom.

2.2. Tailings. Tailings were sampled from a copper mine in China, and the average specific gravity is 2.966 , fine particle size of $-74 \mu \mathrm{m}$ proportion is $87.4 \%$, and the particle size of $-37 \mu \mathrm{m}$ proportion is $68.36 \%$. Particle size distribution indicates that high proportion of fine particles content in the tailings may greatly affect the dewatering performance through gravity sedimentation.

2.3. Mud Bed Sample Preparation. Sampling preparation of mud bed with solid-liquid phase content was fundamental for industrial CT scanning and image analysing; this process is shown in Figure 2.

Before the thickening test, polymer solution was prepared at $0.3 \mathrm{wt} . \%$ concentration. Tailings slurry at $15 \mathrm{wt} . \%$ concentration was held in the tank with a paddle mixer. The thickener was fed with polymer solution and tailings, until the conditions of steady slurry height and underflow concentration were obtained, then the raking was turned off, the supernatant water was discharged, and the rake and columns were disassembled for mud bed sampling. Another batch of test was operated and sampled at the same condition but without raking. The viscosity and solid concentration tests for thickening performance prediction were undertaken at the same time.

The samples of mud bed with raking and without raking were taken in a $10 \mathrm{~mm}$ inner diameter and $100 \mathrm{~mm}$ height Perspex tube inserted into mud bed vertically, during two batches of thickening operations. Then, both sides of the tube were sealed and placed into the liquid nitrogen tank immediately for deep-freezing. After that, these two tubes were kept in a vacuum freeze-drying machine at a pressure of $-96 \mathrm{~Pa}$ and temperature of $-40^{\circ} \mathrm{C}$ for 48 hours. During the process, water has been extracted out after sublimation and in situ structure of aggregate and pore distribution remained.

2.4. Industrial CT Scanning. Due to the advantage of highdensity resolution ratio without tissue overlapping imaging, CT microscopy technique was widely used [18]. After deepfreezing and freeze-drying, the samples have been sent to NANOTOM-160 high-accuracy industrial microscopy CT scanning system individually. The scanning parameters are set at 1000 times magnification and $5 \mu \mathrm{m}$ resolution ratio for each unit, which means the distance of each scanning layer is $5 \mu \mathrm{m}$ and this provides the image of pore size over $5 \mu \mathrm{m}$. In order to avoid microstructure status disarrangement of the periphery and edges of samples when sampling, only take the range of $50 \times 50 \mathrm{~mm}$ in section and $80 \mathrm{~mm}$ in length of the sample for analysis (Figure 3).

\subsection{Reconstruction of Three-Dimensional Images.} According to the obvious differences of the grey level between solid particle and pore, it was very easy to distinguish them from each other by image analysis, just as the great difference of density between them. After eliminating the invalid information via image filtering and noise pixels removing, image binarization processing was able to provide valid information for in situ microstructure.

$3 \mathrm{D}$ contour curves have been built up by connecting the adjacent pixels representing pores from binarized images in three dimensions, which were assembled by several CT 


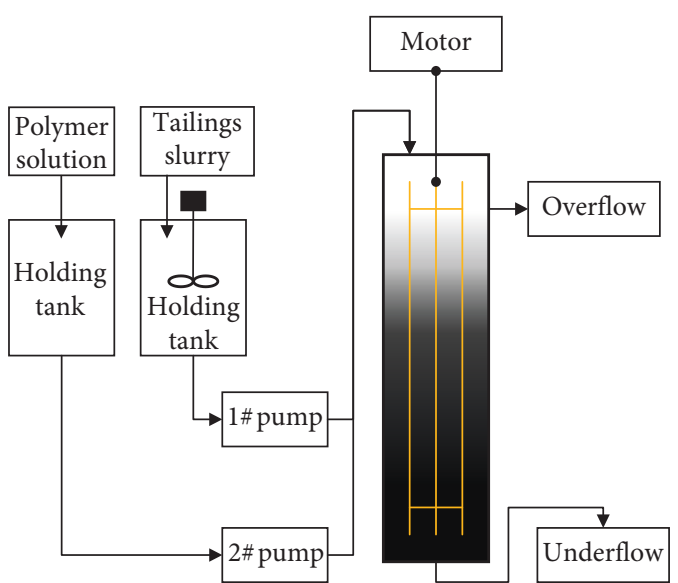

(a)

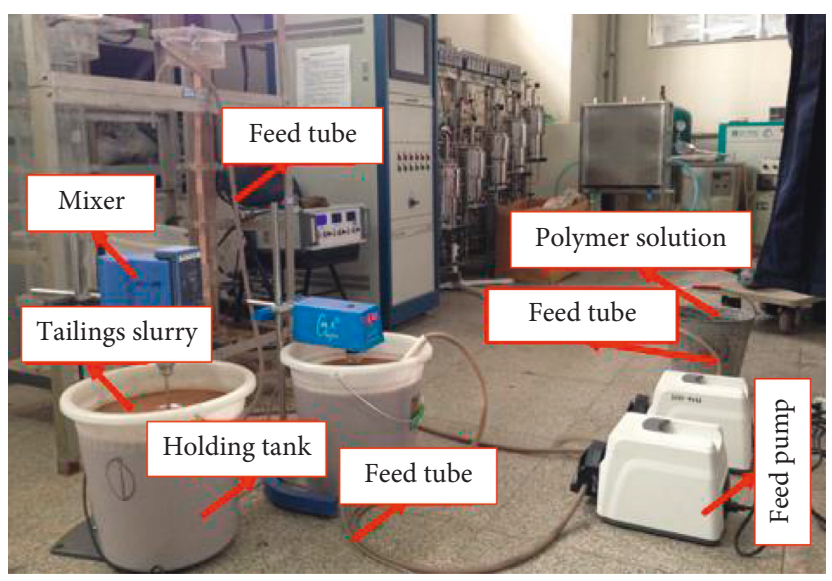

(b)

Figure 1: Pilot thickener test system.

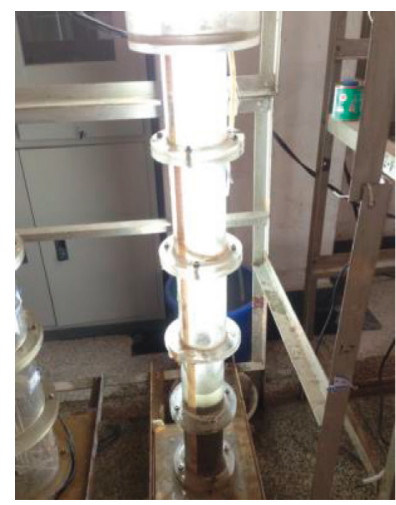

(a)

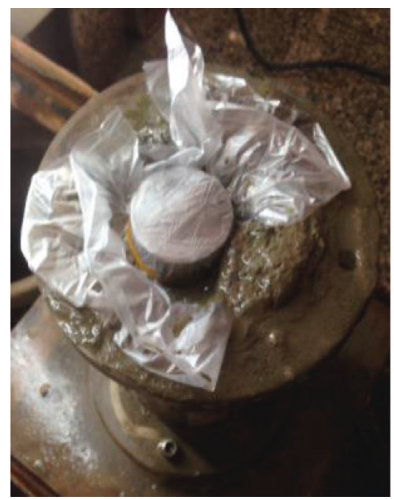

(b)

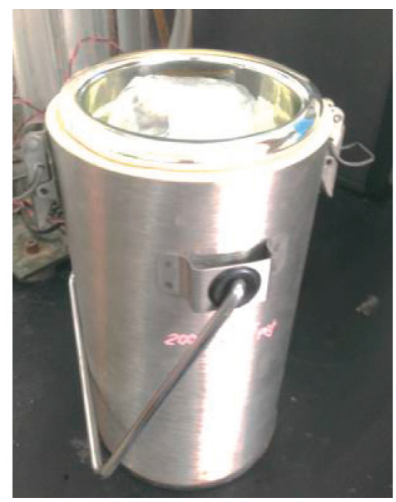

(c)

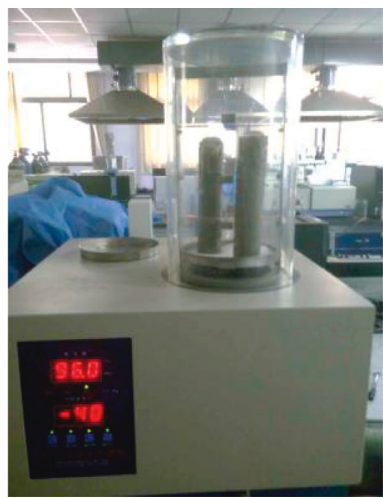

(d)

Figure 2: Mud bed sample preparation. (a) Thickening operation. (b) Sampling. (c) Deep-freezing. (d) Freeze-drying.

scanning images at the same interval. Consequently, the contour curves consist of the surfaces and 3D model of pore structure. Then, Matlab is used to calculate the particle and voids distribution on sections.

\section{Results and Discussion}

3.1. Channelling Development. Flocculation improved the sedimentation of tailings particles during thickening processes [19]. Large and porous aggregates of tailings settled at the bottom of the thickener, accumulated, and formed as networked mud bed, after the solid concentration went beyond the gel point [20]. At the beginning, networked mud bed bears the loading of upside slurry under temporary balance of total stress and the pore pressure of the fluid [21] and water was trapped inside or between the pore of aggregates. When tailings feed was continuous, the gravity of slurry increased and gentle shear stress induced by raking could break the fragile structure of aggregates easily [22]. Isolated pores inside or between aggregates were connected to each other by throat and then formed as larger voids or tortuous channels (Figure 4).
Channels arise as vertical tortuous paths to transmit bound water of aggregates and dissipate the pore pressure, and at the same time, the hydrodynamic drag force caused by the particles decreased as the aggregates broke up and channels formed [23]. After bound water has been squeezed upward to the interface of mud bed and supernatant, aggregates reconstructed by smaller and denser aggregates filled into the voids of escaped bound water and the interval of aggregates decreased (Figure 5). Meanwhile, the structure of pore connection or channels was extremely unstable; this kind of temporary microstructure periodicity generated, grown, and closed as low shear ratio of periodicity raking. The development of channels depends on the duration time of shear and the distance from the interface of mud bed and supernatant.

3.2. Channelling Structures. Channel formed as aggregate densification occurred, and it consists of voids and connecting throats [24]. Pores are the space surrounded by particles or aggregates, and the throat is the narrow access connecting two particles or aggregates. Each throat connected two voids, 

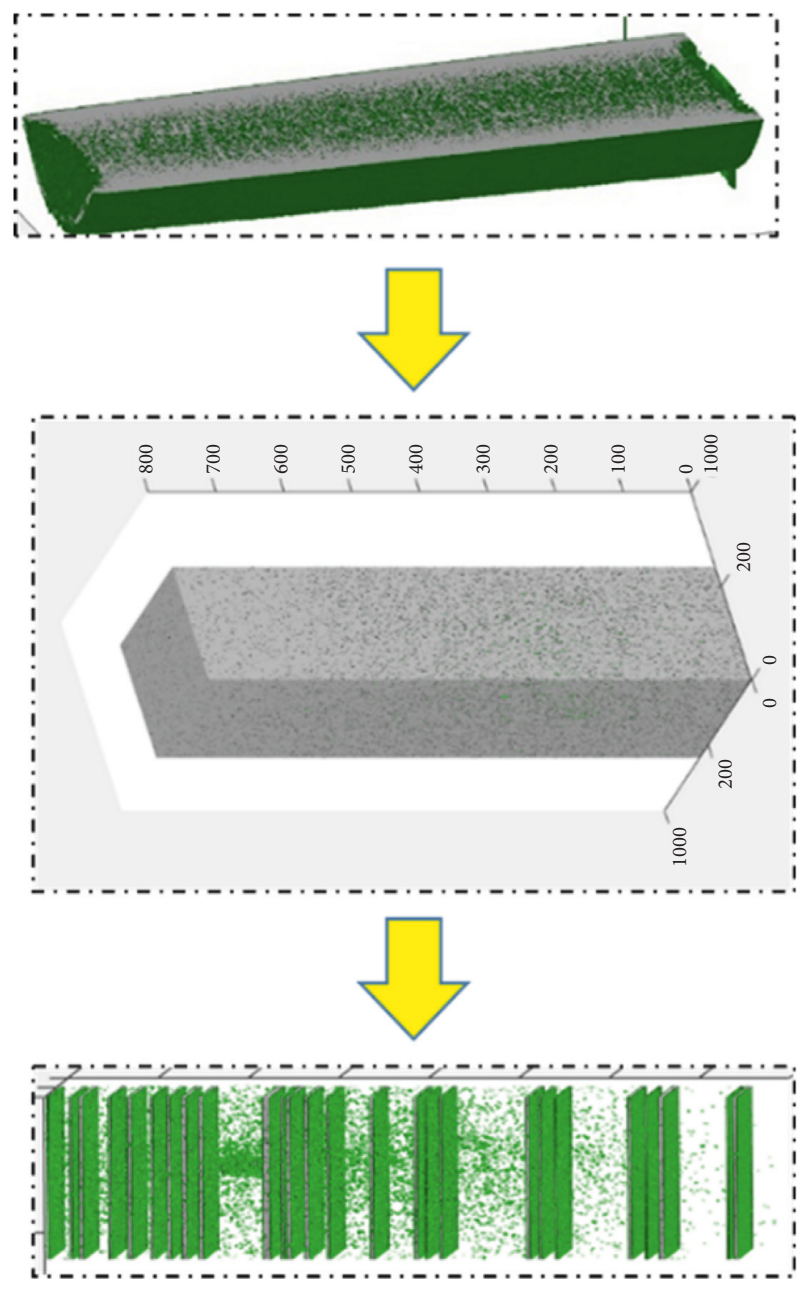

FIgURE 3: Invalid area and effective area extraction.

while the voids were connected with at least three throats. According to the different structures of pore and throat [25], channels of mud bed during thickening were divided into 5 types (Figure 6): columnar throat channel, acicular throat channel, flaky throat channel, curved flaky throat channel, and capillary fasciculate throat channel.

Based on the feature of hydraulic conductivity, channels could be classified as effective and ineffective channels. The effective channels consist of large-sized pores connected with a large throat; these channels accelerate bound water flow upward, which dissipates pore pressure effectively. Meanwhile, the ineffective channels consist of the small-size pores connected with capillary throat, which means even the aggregate with high porosity and the bound water would be trapped in the isolated pore because of ineffective water flow paths. Depending on mechanical equilibrium differences, the effective channels likely occurred at the early stage of raking or at a higher position of mud bed and the ineffective channels occurred after sufficient shear or at the bottom of mud bed.

3.3. Fractal Characteristics of Pore Structure. Fractal geometry is the theory for studying irregular curves with self- similarity and irregular patterns with self-reversal; it is an effective tool for the microstructure research. The fractal features of materials can be characterized by fractal dimension. There are many definitions of dimensions, including Hausdorff dimension, box-counting dimension, and space-filling dimension [26]. Among them, the boxcounting dimension is easy to programmatically calculate and has been widely used in research. The pixel count method was used to estimate the box-counting dimension of the binarized images of the mud bed microstructure.

The image is binarized, pixels of the image are separated into black and white, and the number of rows and columns corresponding to the number of rows and columns of the binary image is obtained. Then, the data were divided into several blocks in order, and the number of rows and columns of each block is $k$, the number of all those blocks containing 0 (or 1) was denoted as $N_{\delta k}$ (abbreviated as $N_{k}$ ), usually $k=1,2,4, \ldots, 2^{i}$, the size of $2^{i}$ pixel points was divided into side blocks, and the number of box count was $N_{1}, N_{2}, N_{4}, \ldots, N_{2^{i}}$. The size of the pixel $\delta$ was calculated as

$$
\delta=\frac{\text { length of image }}{\text { pixel counts of a row in image }} \text {. }
$$

Therefore, the length of the block containing $k$ pixels is $\delta_{k}=k \delta, k=1,2,4, \ldots, 2^{i}$. Since $\delta$ is a constant for a specific image, $\delta_{k}$ can be substituted with $k$ in calculation. In the double logarithmic coordinate, the data points $\left(\log \delta_{k}, \log \right.$ $N_{k}$ ) were fitted by straight line by the least-squares method, where $k=1,2,4, \ldots, 2^{i}$. The negative value $D_{\mathrm{b}}$ of the slope of the obtained straight line was the box-count fractal dimension of the pore:

$$
D_{\mathrm{b}}=-\frac{\log N_{k}}{\log \left(\delta_{k}\right)}
$$

Images of sheared mud bed sample have been binarized by denoising, histogram equalization, and histogram normalization; the box-count dimension in different bed heights is calculated as the size of block $\delta_{k}$ is $8 \sim 100$ pixels length (Figure 7).

The maximum box-count dimension $D_{\mathrm{bmax}}$ of the aggregate at the top of the sheared mud bed sample is 1.41. As the mud bed height gets closer to the bottom of the thickener, the box-count dimension $D_{\mathrm{b}}$ gradually increased to 1.57 and eventually approached a constant value (Figure 8 ). The box-count dimension $D_{\mathrm{b}}$ was the exponential function of mud bed height. Aggregate fractal dimension of sheared mud bed sample was increased as the aggregate structure broke up and solid effective strength increased, which caused the void ratio and pore size decrease and the tailing solid concentration increase. The aggregates densification and pores deformation were obviously affected by the shear of rake in the vertical direction.

3.4. Void Ratio. We can assume that the process of aggregate structure break and reconstruction could be profiled as the variation of void ratio, pore amount, and pore size, when aggregate densification and channelling occur. Based on binarized CT scanning images, void ratio between and inside 


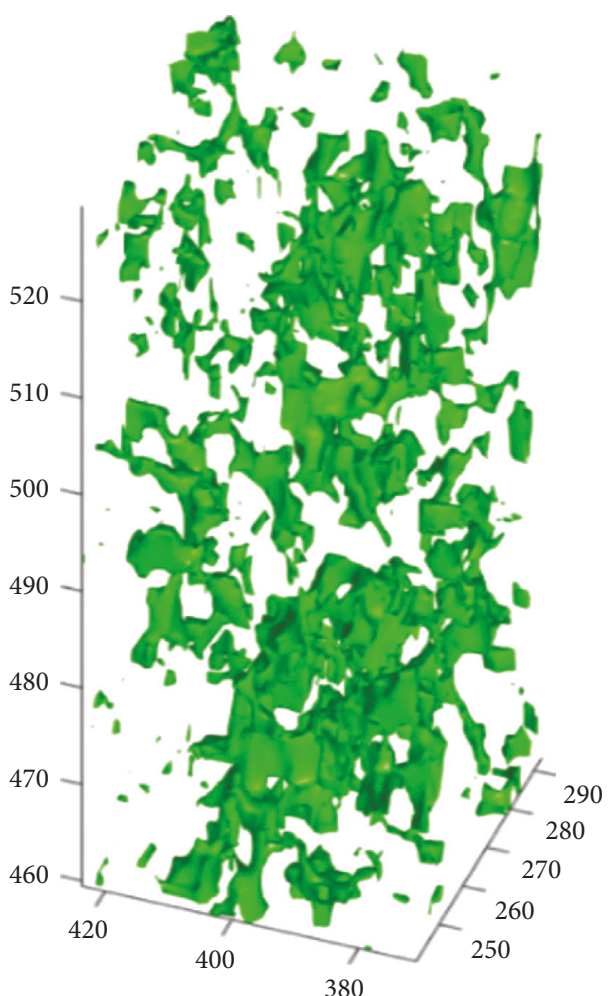

(a)

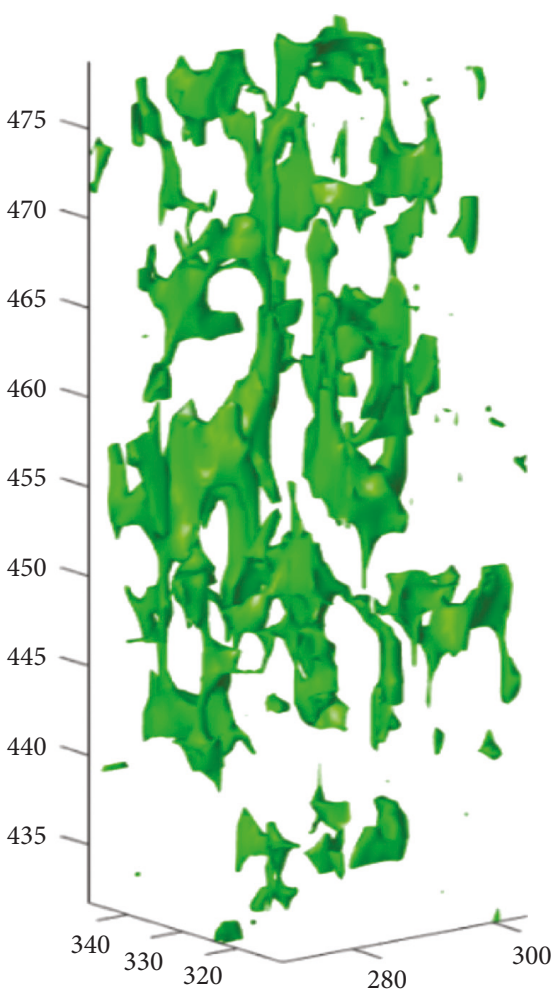

(b)

Figure 4: Pore distribution and channel development affected by raking.

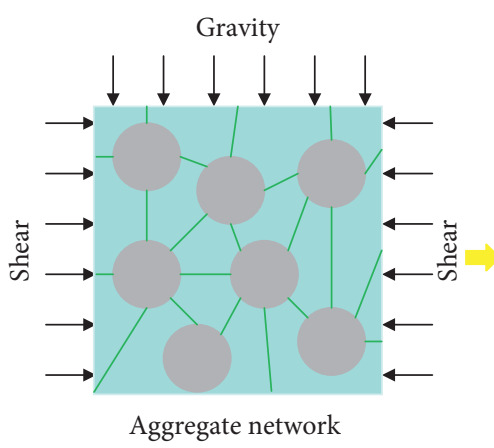

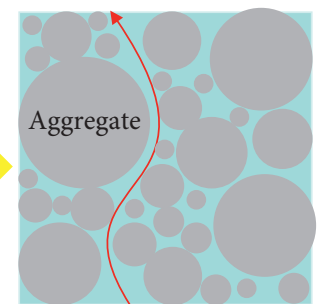

Channel formation

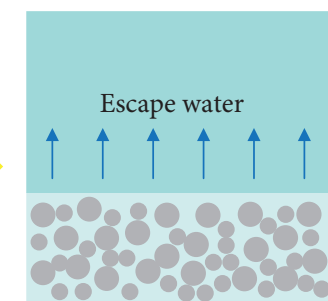

Aggregate densification

FIgURE 5: Scheme of aggregate densification and channel formation.

of aggregates was calculated as the pore pixels area divided by total pixels area of the image, which represents the surface void ratio $(n)$ of a certain layer of sample:

$$
n=\frac{\text { pore pixels amount }}{\text { image total pixels amount }} \times 100 \% \text {. }
$$

Void ratio increase with the height (distance from the bottom of thickener) in sheared and unsheared samples (Figure 9), which means the mud bed was closer to the underflow discharge outlet and of lower permeability, but with a higher solid concentration. The void ratio of the unsheared sample varied from $44.7 \%$ to $49.6 \%$, and the average void ratio was $47.6 \%$, while void ratio varied from $37.8 \%$ to $60.7 \%$ and the average void ratio was $40.9 \%$ in the sheared sample. Meanwhile, the pore amount of the sheared and unsheared sample was almost constant, average pore amount was 8036 in the unsheared sample, and average pore amount was 7968 in sheared sample. Raking affects aggregates structure by decrease in the pore size.

The pore size morphological opening operations are performed on binarized images, based on increasing the size of the disc structure element. Each calculation of the erosion algorithm and dilation has taken into account the sum total of each pixel. Because the pore shape varied from each other, only the maximum tangent equivalent diameter was obtained through calculate operation, which depends on void size.

Figure 10 shows pore size distribution variation caused by shear of raking. The largest pore diameter of the unsheared sample is $464 \mu \mathrm{m}$, and the proportion of pore diameter larger than $45 \mu \mathrm{m}$ is $81.2 \%$, compared to the sheared sample, with largest pore diameter being $206 \mu \mathrm{m}$ and the proportion of pore diameter larger than $45 \mu \mathrm{m}$ being 


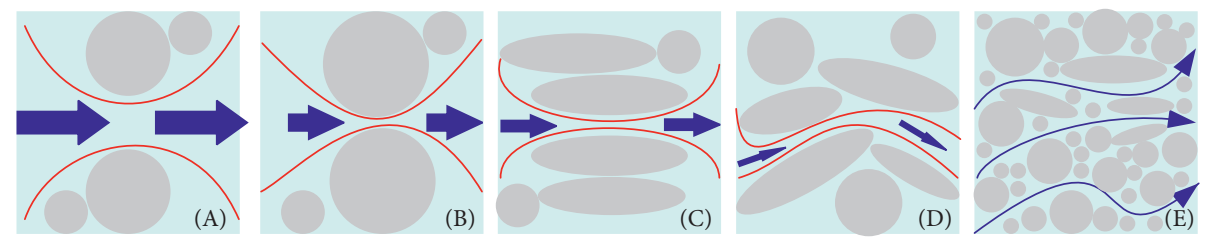

(a)
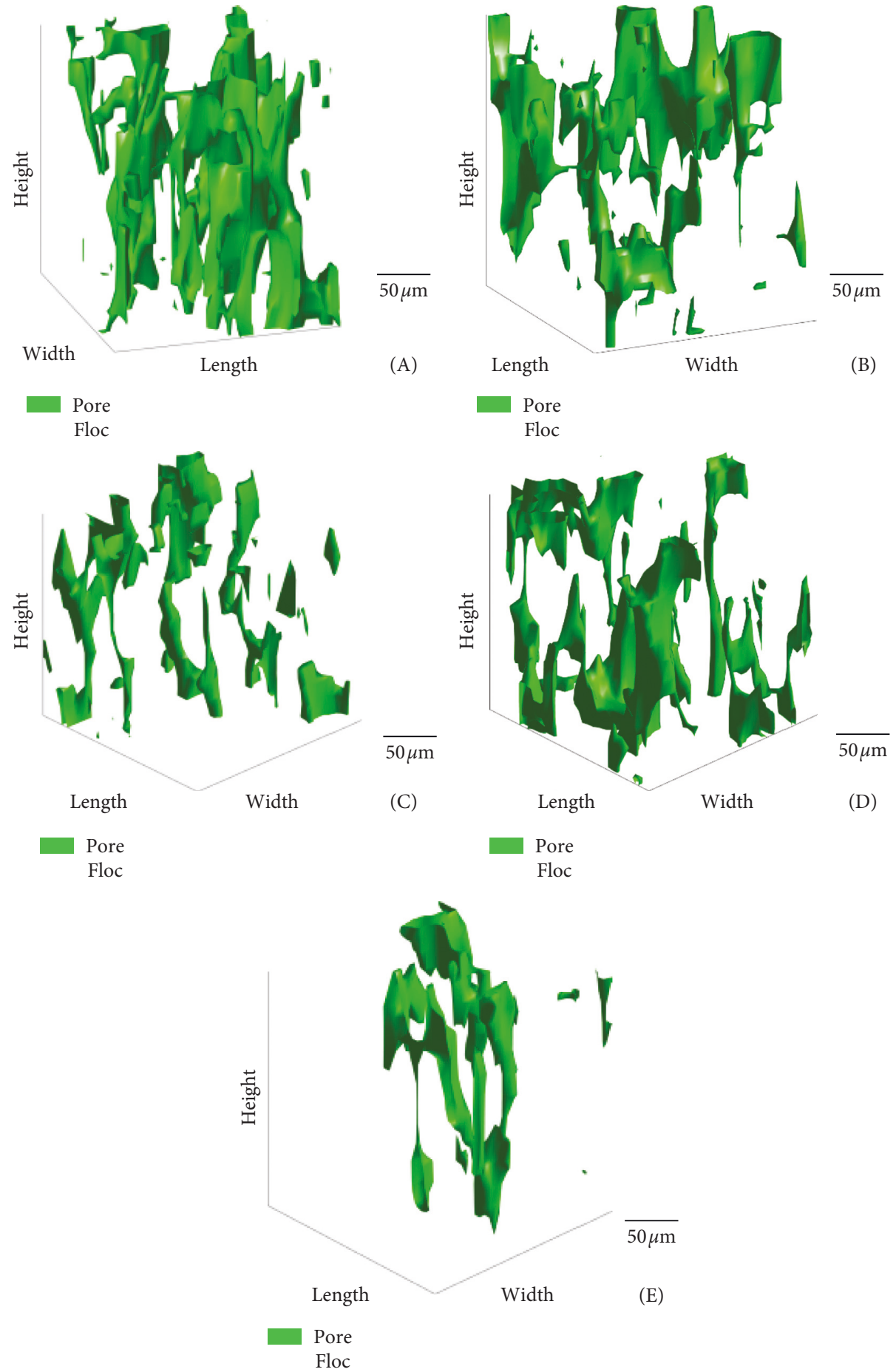

(b)

Figure 6: (a) Scheme and (b) three-dimensional reconstruction model of channel classification. (A) Columnar throat channel. (B) Acicular throat channel. (C) Flaky throat channel. (D) Curved flaky throat channel. (E) Capillary fasciculate throat channel. 


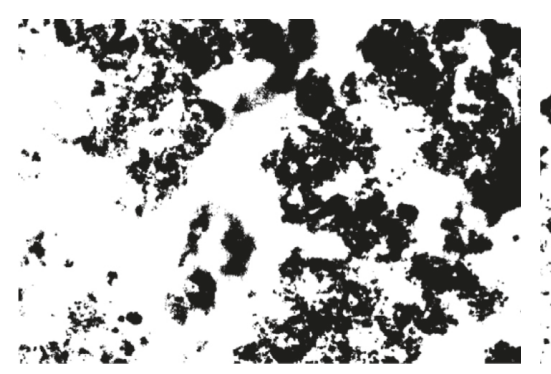

(A) $75 \mathrm{~mm}$

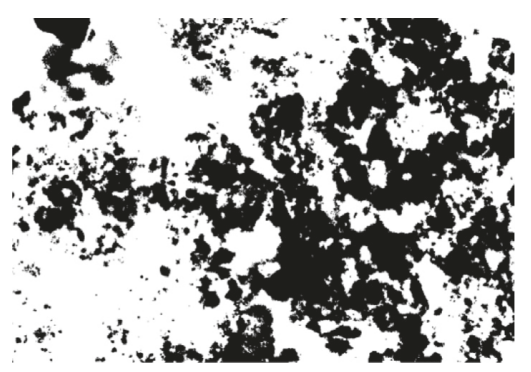

(B) $45 \mathrm{~mm}$

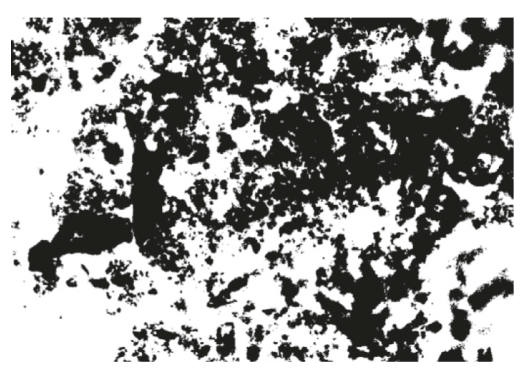

(C) $15 \mathrm{~mm}$

(a)

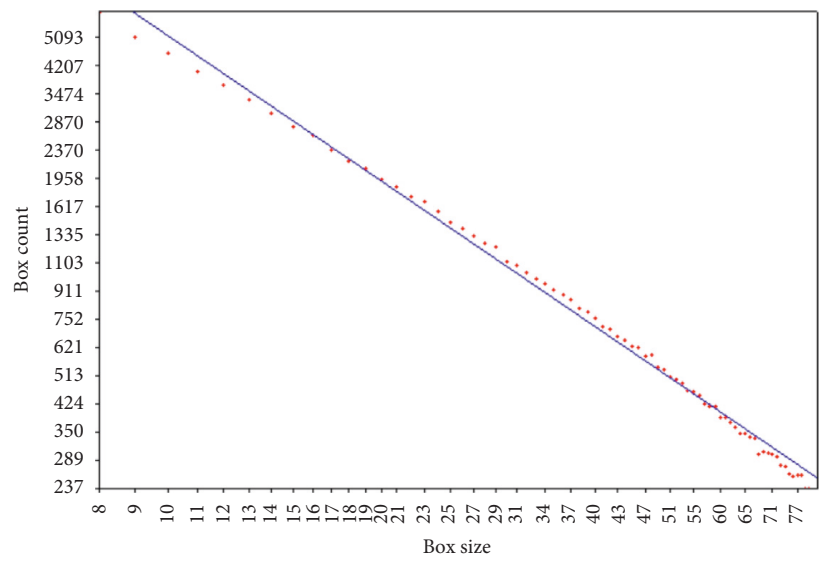

(A) Box-counting $(75 \mathrm{~mm})$

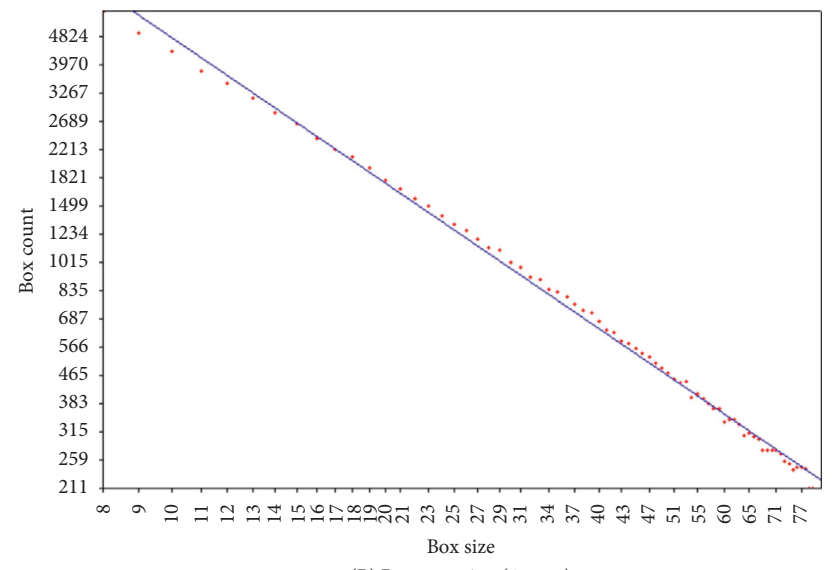

(B) Box-counting $(45 \mathrm{~mm})$

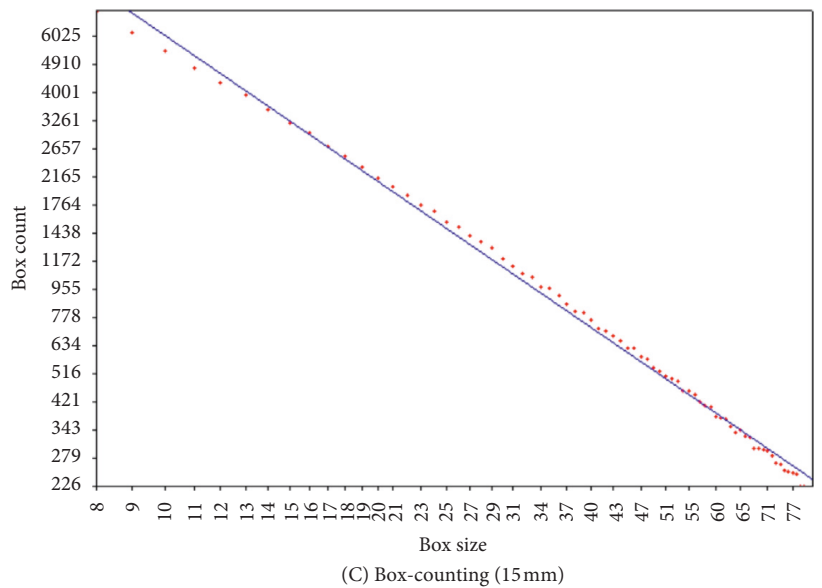

(b)

Figure 7: Aggregate fractal dimension of mud bed at different heights.

$57.9 \%$. Consequently, the pore size between and inside of aggregates decreased, trapped water has been squeezed out from isolated pore, and then solid concentration increased, due to aggregates reconstruction after raking. The void ratio and pore size results correspond to the pore dimension.

3.5. Permeability. Permeability $k(\phi)$ is the measurement of how easily water flows through mud bed during thickening, indicating the dewatering extent of tailings [27]. Kozeny introduced the relation between permeability and the properties of the porous medium and it was later modified by Carman, and it is commonly known as the KozenyCarman equation [28]. Based on the basic theory, many other empirical equations focus on the relation of permeability and particle size [29]. Besides that, Istomina provided another prediction for permeability from the void ratio instead of particle size [30]. The function between permeability and pore size was set up according to the void ratio and pore size distribution parameters calculation:

$$
k=\frac{g n d_{\mathrm{p}}^{2}}{96 \mu_{\mathrm{w}}},
$$

where $k$ is the permeability, $g$ is the gravity acceleration, $d_{\mathrm{p}}$ is the average pore diameter, $n$ is the void ratio, and $\mu_{\mathrm{w}}$ is the viscosity of water. The average permeability decreased from $6.35 E-4 \mathrm{~mm} / \mathrm{s}$ to $7.65 E-5 \mathrm{~mm} / \mathrm{s}$ in the sheared sample, and 


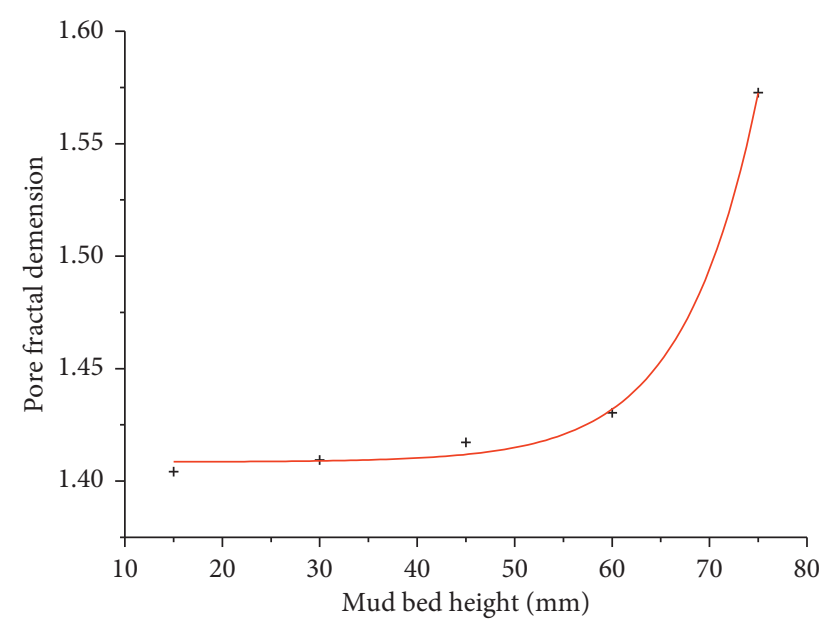

Figure 8: Pore fractal dimension of mud bed at different heights.

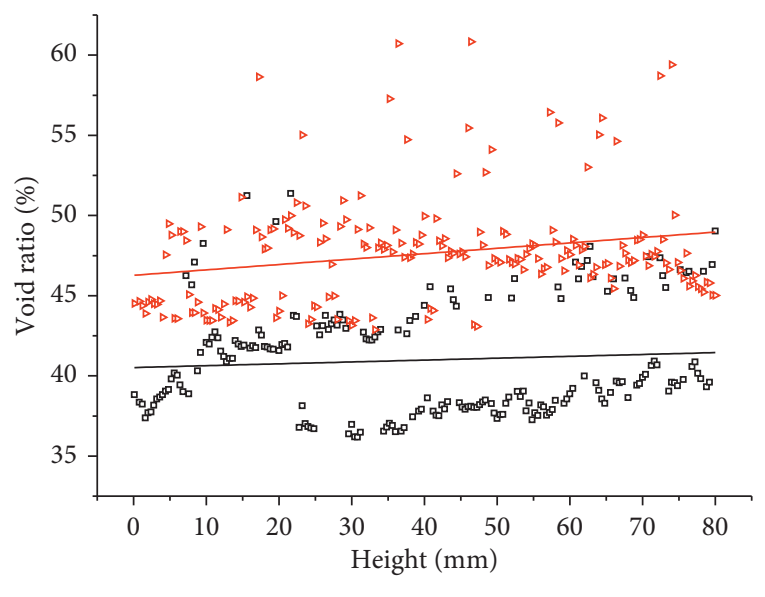

- Sheared

$\checkmark$ Unsheared

FIgURE 9: Void ratio variation with height.

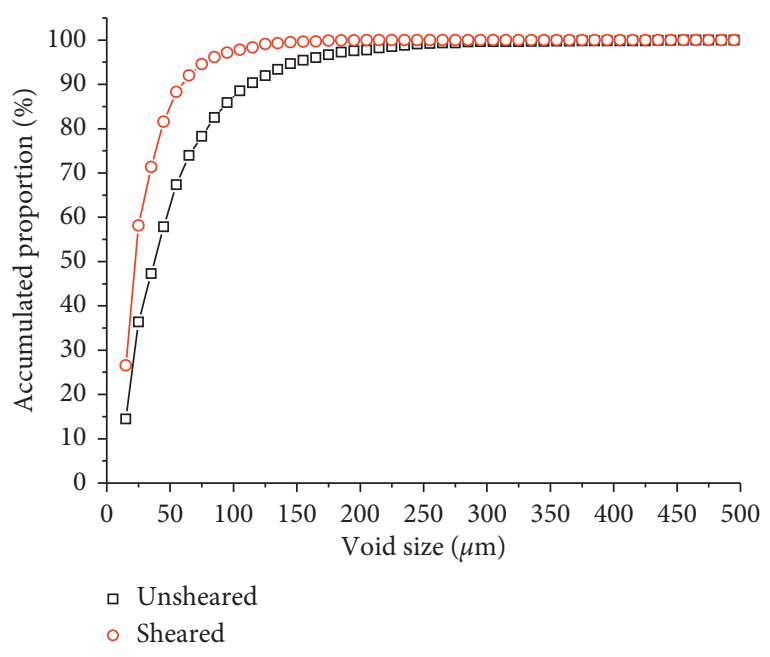

FIgURE 10: Average pore size distribution with height.

it changed from $1.21 E-3 \mathrm{~mm} / \mathrm{s}$ to $1.51 E-4 \mathrm{~mm} / \mathrm{s}$ in the unsheared sample; permeability was correlated as the exponential function of void ratio, as shown in Figure 11.

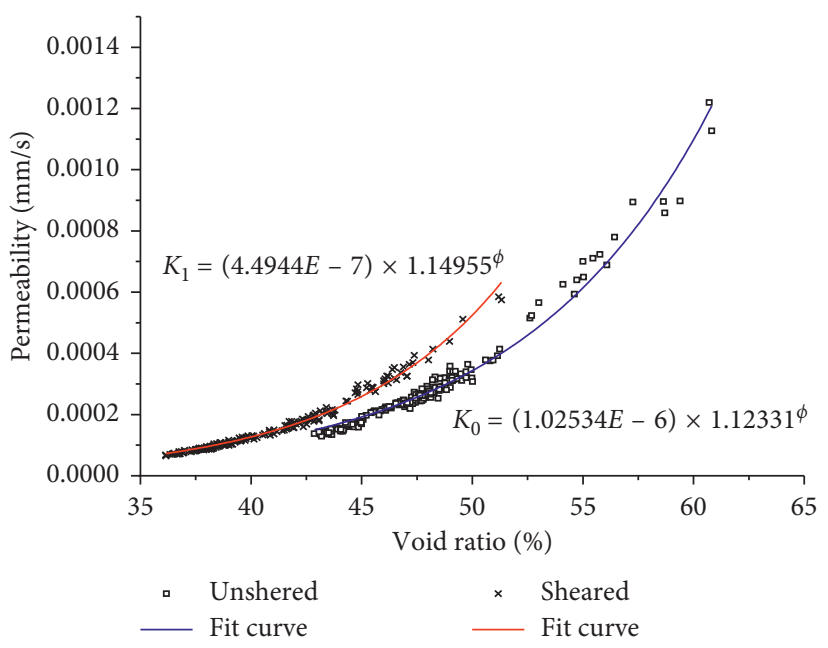

FIgURE 11: Correlation of permeability and void ratio.

Permeability decreased as the void ratio reduced, indicating the hydrodynamic resistance to water flow through tailings suspension increased.

3.6. Thickening Performance Enhancement. Tailings dewatering extent was quantified as solid flux, which is the product of solids fraction and solids settling velocity [31]. Solids flux asymptotes to zero, as solid particle settling through densified mud bed was strongly hindered by other particles [32].

During dewatering of thickening, water flows through the porous aggregates constituting the sediment; the solidfluid interaction force during consolidation was the function of bed permeability and water viscosity, which represents Darcy's equation [33]:

$$
m_{\mathrm{d}}=\frac{\mu}{k(\phi)}(1-\phi)^{2} v_{\mathrm{r}}
$$

where $m_{\mathrm{d}}$ is the solid-fluid interaction force, $\phi$ is the solid volume fraction, $k(\phi)$ is the permeability of mud bed slurry, and $v_{\mathrm{r}}$ is the relative solid-fluid velocity. Replacing $m_{\mathrm{d}}$ and $v_{\mathrm{r}}$ as constitutive equations of dynamic thickening process, we get

$$
\begin{aligned}
\frac{\partial \sigma_{\mathrm{e}}}{\partial z} & =-\Delta \rho g \phi+\frac{m_{\mathrm{d}}}{1-\phi}, \\
v_{\mathrm{r}} & =-\frac{k(\phi)}{\mu} \times \frac{\Delta \rho g \phi}{1-\phi} \times\left(1+\frac{\sigma_{\mathrm{e}}^{\prime}(\phi)}{\Delta \rho g \phi} \frac{\partial(\phi)}{\partial z}\right),
\end{aligned}
$$

where $g$ is the gravity acceleration, $\sigma_{\mathrm{e}}$ is the effective solid stress, $\mu$ is the viscosity of slurry, and $\Delta \rho$ is the density difference of tailings and water.

Substituting the equations, the solid flux density function for the whole range of concentration is as follows [34]:

$$
f_{b k}(\phi)=\left\{\begin{array}{cc}
-\frac{\Delta \rho g \phi^{2}(1-\phi)^{2}}{\mu k(\phi)}, & \text { for } \phi<\phi_{g}, \\
-\frac{k(\phi)}{\mu} \Delta \rho g \phi^{2}, & \text { for } \phi<\phi_{g},
\end{array}\right.
$$




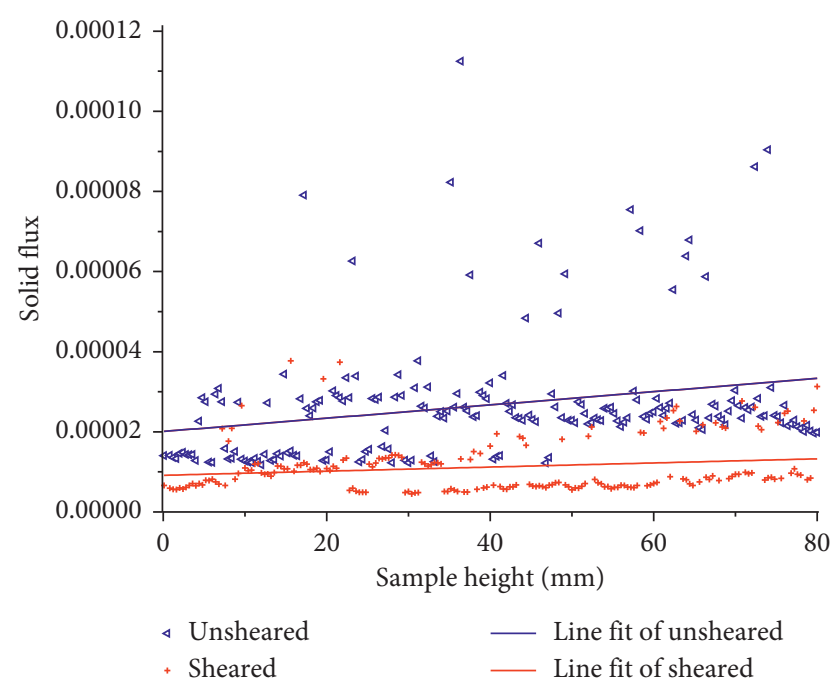

FIGURE 12: Solid fluxes of sheared and unsheared samples.

where $\phi$ is the solid volume fraction, $f_{b k}(\phi)$ is the solid flux, $k(\phi)$ is the permeability of mud bed slurry, $\Delta \rho$ is the density difference of tailings and water, $\mu$ is the viscosity of slurry, and $g$ is the gravity acceleration.

Introducing equation (4) into equation (7), the solid flux of mud bed at the solid concentration beyond the gel point $\left(\phi \geq \phi_{g}\right)$ was obtained from the void ratio, pore size, rheology, and solid concentration profile:

$$
f_{b k}(\phi)=\frac{g n d_{\mathrm{p}}^{2}}{96} \times \frac{\Delta \rho \phi^{2} g}{\mu} .
$$

Solid flux decreased after sufficient raking, and the average solid particles flux of the unsheared and sheared samples was $2.67 E-5$ and $1.12 E-5$ (Figure 12). The thickener dewatering performance enhancement factor was defined as $\mathrm{PE}=$ solid flux of unsheared condition divided by solid flux of sheared condition; in this study, $\mathrm{PE}=3.05$. The performance enhancement significantly increased with the shear of raking, as the consequence of aggregate densification and channelling, and shear effects on aggregates reconstruction and channelling enhanced solid concentration of mud bed slurry during thickening.

\section{Conclusions}

Aggregate structures were broken under gravity coupled with shear stress of raking, and the isolated pores inside and between aggregates were connected and formed as channels. According to the shape and size of the throat which connected with the pore, channelling patterns have been divided into 5 types, which are columnar throat channel, acicular throat channel, flaky throat channel, curved flaky throat channel, and fasciculate capillary throat channel.

Box-count dimension $D_{\mathrm{b}}$ of aggregate in the sheared mud bed sample was the exponential function of mud bed height. The maximum box-count dimension of the aggregate at the bottom of the sheared mud bed sample is 1.57 and eventually approached to a constant value as the shear time extended. When the mud bed height gets closer to the interface of solid-liquid, the box-count dimension $D_{\mathrm{b}}$ gradually decreased to 1.41 .

Isolated pore size and void ratio were significantly affected by shear of raking, and the proportion of pore diameter larger than $45 \mu \mathrm{m}$ from $81.2 \%$ decreased to $57.9 \%$, while the void ratio reduced by $8.7 \%$. Permeability has been set up as the exponential function of void ratio, which decreased by $58.93 \%$ compared to the unsheared sample.

The average dewatering performance enhancement factor $\mathrm{PE}=3.05$ has been predicted, according to the solid flux of unsheared and sheared samples. Raking affected the aggregates densification and channelling development, which contributes to solid flux decrease and concentration increase during the mud bed thickening.

\section{Data Availability}

All of the underlying data related to this submission were obtained from our research.

\section{Conflicts of Interest}

The authors declare that they have no conflicts of interest.

\section{Acknowledgments}

This work was financially supported by the National Key Technologies R\&D Program for the 13th Five-Year Plan (2017YFC0602903), the National Natural Science Foundation of China (51574013 and 51674012), and the National Natural Science Foundation Youth Fund Project of China (51704094).

\section{References}

[1] Y. Erol, "Advances in reducing large volumes of environmentally harmful mine waste rocks and tailings introduction," Gospodarka Surowcami Mineralnymi, vol. 27, no. 2, pp. 89112, 2011.

[2] P. C. Larrauri and U. Lall, "Tailings dams failures: updated statistical model for discharge volume and runout," Environments, vol. 5, no. 2, p. 28, 2018.

[3] H. Lu, C. Qi, Q. Chen et al., "A new procedure for recycling waste tailings as cemented paste backfill to underground stopes and open pits," Journal of Cleaner Production, vol. 188, pp. 601-612, 2018.

[4] A. Blanco, A. Lloret, J. Carrera, S Olivella, and ., "Thermohydraulic behaviour of the vadose zone in sulphide tailings at Iberian Pyrite Belt: waste characterization, monitoring and modelling," Engineering Geology, vol. 165, pp. 154-170, 2013.

[5] M. Benzaazoua, M. Fall, and T. Belem, "A contribution to understanding the hardening process of cemented pastefill," Minerals Engineering, vol. 17, no. 2, pp. 141-152, 2004.

[6] A. Ghirian and M. Fall, "Long-term coupled behaviour of cemented paste backfill in load cell experiments," Geomechanics and Geoengineering, vol. 11, no. 4, pp. 237-251, 2016.

[7] P. Sandeep, D. Debasis, and T. Sreenivas, "Variability in rheology of cemented paste backfill with hydration age, binder and superplasticizer dosages," Advanced Powder Technology, vol. 29, no. 9, pp. 2211-2220, 2018. 
[8] H. Li, H. J. Wang, A. X. Wu, and H. Z. Jiao, "Pressure rake analysis of deep cone thickeners based on tailings' settlement and rheological characteristics," Journal of University of Science \& Technology Beijing, vol. 35, no. 12, pp. 1553-1558, 2013.

[9] S. P. Usher and P. J. Scales, "Steady state thickener modelling from the compressive yield stress and hindered settling function," Chemical Engineering Journal, vol. 111, no. 2-3, pp. 253-261, 2005.

[10] B. R. Gladman, M. Rudman, and P. J. Scales, "Experimental validation of a 1-D continuous thickening model using a pilot column," Chemical Engineering Science, vol. 65, no. 13, pp. 3937-3946, 2010.

[11] A. N. Papanicolaou and A. R. Maxwell, "Methodological considerations for studying self-weight fluidization in a sedimentation column," International Journal of Mineral Processing, vol. 78, no. 3, pp. 140-152, 2006.

[12] T. S. Steenhuis, C. E. Baver, B. Hasanpour, C. R. Stoof, D. A. DiCarlo, and J. S. Selker, "Pore scale consideration in unstable gravity driven finger flow," Water Resources Research, vol. 49, no. 11, pp. 7815-7819, 2013.

[13] E. M. Tory, R. Bürger, F. Concha, and M. C. Bustos, Sedimentation and Thickening: Phenomenological Foundation and Mathematical Theory, Springer Science \& Business Media, Berlin, Germany, 2013.

[14] J. O’Donnell and N. Bayrak, Review of Channelling in Batch Sedimentation, Institution of Engineers, Australia, Australia, 2004.

[15] R. G. Holdich and G. Butt, "Experimental and numerical analysis of a sedimentation forming compressible compacts," Separation Science and Technology, vol. 32, no. 13, pp. 21492171, 1997.

[16] G. G. Glasrud, R. C. Navarrete, L. E. Scriven, and C. W. Macosko, "Settling behaviors of iron-oxide suspension," AIChE Journal, vol. 39, no. 4, pp. 560-568, 2010.

[17] S. Nam, M. Gutierrez, and P. Diplas, "Channeling during settling and self-weight consolidation of cohesive sediments," Canadian Geotechnical Journal, vol. 45, no. 6, pp. 867-876, 2008.

[18] S. M. Bello, I. De Groote, and G. Delbarre, "Application of 3dimensional microscopy and micro-CT scanning to the analysis of magdalenian portable art on bone and antler," Journal of Archaeological Science, vol. 40, no. 5, pp. 24642476, 2013.

[19] N. Alam, O. Ozdemir, M. A. Hampton, and A. V. Nguyen, "Dewatering of coal plant tailings: flocculation followed by filtration," Fuel, vol. 90, no. 1, pp. 26-35, 2011.

[20] B. R. Gladman, M. Rudman, and P. J. Scales, "The effect of shear on gravity thickening: pilot scale modelling," Chemical Engineering Science, vol. 65, no. 14, pp. 4293-4301, 2010.

[21] S. P. Usher, R. Spehar, and P. J. Scales, "Theoretical analysis of aggregate densification: impact on thickener performance," Chemical Engineering Journal, vol. 151, no. 1-3, pp. 202-208, 2009.

[22] B. B. G. van Deventer, S. P. Usher, A. Kumar, M. Rudman, and P. J. Scales, "Aggregate densification and batch settling," Chemical Engineering Journal, vol. 171, no. 1, pp. 141-151, 2011.

[23] J. B. Farrow, R. R. M. Johnston, K. Simic, and J. D. Swift, "Consolidation and aggregate densification during gravity thickening," Chemical Engineering Journal, vol. 80, no. 1-3, pp. 141-148, 2000.

[24] G. M. Laudone, C. M. Gribble, and G. P. Matthews, "Characterisation of the porous structure of gilsocarbon graphite using pycnometry, cyclic porosimetry and void-network modeling," Carbon, vol. 73, no. 1, pp. 61-70, 2014.
[25] Z. T. Luo and Y. C. H. Wang, Pore Structure of Oil and Gas Reservoirs, Science Press, Beijing, China, 1986.

[26] M. Fernández-Martínez and M. A. Sánchez-Granero, "A new fractal dimension for curves based on fractal structures," Topology and Its Applications, vol. 203, no. 15, pp. 108-124, 2016.

[27] R. Spehar, A. Kiviti-Manor, P. Fawell et al., "Aggregate densification in the thickening of flocculated suspensions in an un-networked bed," Chemical Engineering Science, vol. 122, pp. 585-595, 2015.

[28] J. Mortensen, F. Engstrom, and I. Lind, "The relation among porosity, permeability, and specific surface of chalk from the gorm field, Danish North Sea," SPE Reservoir Evaluation \& Engineering, vol. 1, no. 3, pp. 245-251, 1998.

[29] S. Taheri, S. Ghomeshi, and A. Kantzas, "Permeability calculations in unconsolidated homogeneous sands," Powder Technology, vol. 321, pp. 380-389, 2017.

[30] J. Odong, "Evaluation of empirical formulae for determination of hydraulic conductivity based on grain-size analysis," Journal of American Science, vol. 3, no. 3, pp. 5460, 2007.

[31] P. Grassia, S. P. Usher, and P. J. Scales, "Closed-form solutions for batch settling height from model settling flux functions," Chemical Engineering Science, vol. 66, no. 5, pp. 964-972, 2011.

[32] Q. G. Guo, Engineering Characteristics and Application of Coarse-Grained Soil, Yellow River Water Conservancy Press, Zhengzhou, China, 1999.

[33] C. A. Fernando, "Solid-liquid separation in the mining industry," Fluid Mechanics \& Its Applications, vol. 105, 2014.

[34] S. Diehl, "Estimation of the batch-settling flux function for an ideal suspension from only two experiments," Chemical Engineering Science, vol. 62, no. 17, pp. 4589-4601, 2007. 


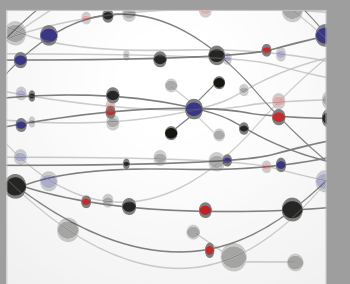

The Scientific World Journal
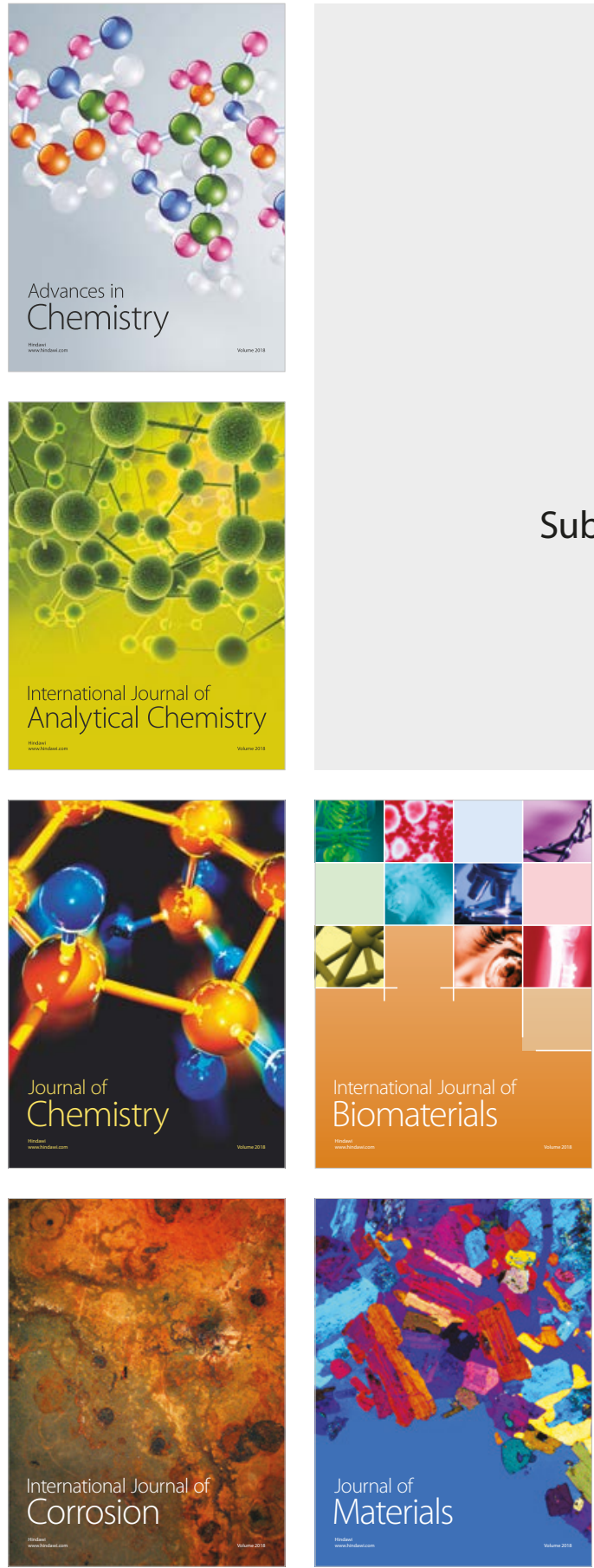

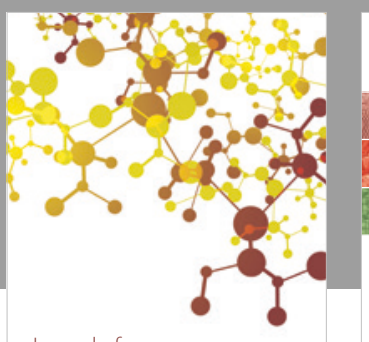

Journal of

Applied Chemistry
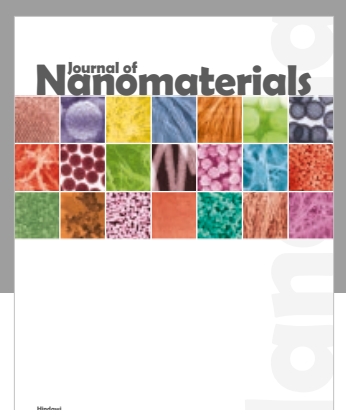

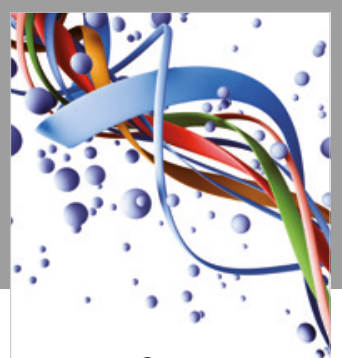

Scientifica

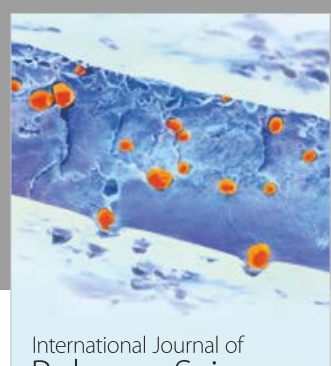

Polymer Science

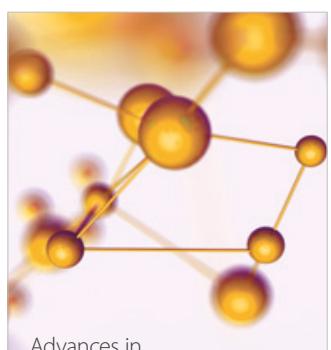

Physical Chemistry
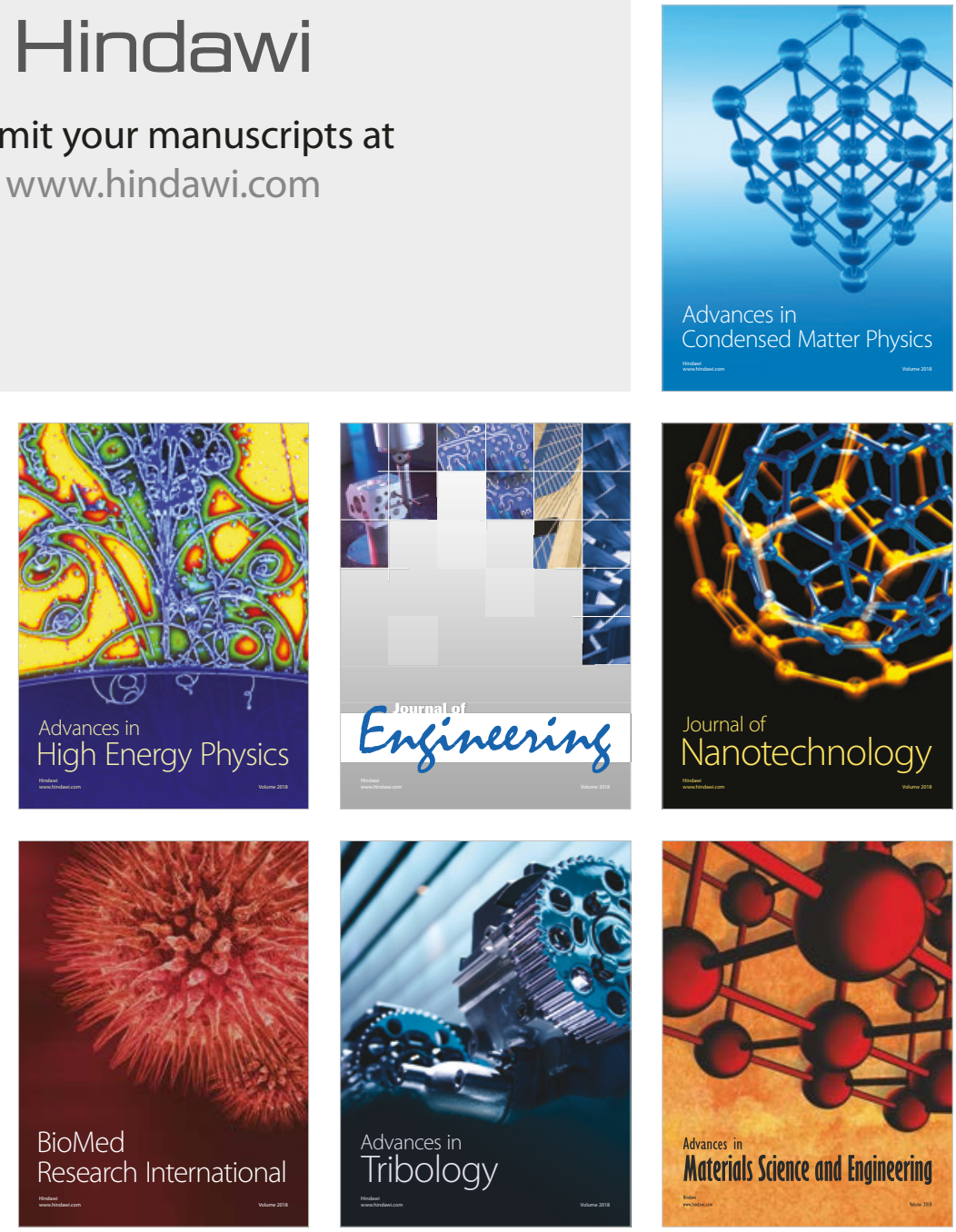